\title{
La Pediatría argentina en los próximos años
}

\section{Argentine pediatrics in the coming years}

La Pediatría siempre se ha caracterizado por el abordaje biopsicosocial de todas las problemáticas vinculadas a la niñez y la adolescencia.

La atención de nuestros pacientes pediátricos, con esta mirada ampliada, lleva implícita la necesidad de involucrarnos en los determinantes sociales del proceso salud-enfermedad, entiende su bienestar no solamente como la ausencia de enfermedad, sino que tiene como meta, por el contrario, que niños, niñas y adolescentes sean capaces de alcanzar sus máximas potencialidades de crecimiento y desarrollo, en un ambiente de contención, afecto y educación de calidad.

En los próximos años los pediatras nos veremos afrontando serias consecuencias sanitarias, producto del deterioro económico y social generado por la pandemia por SARS$\mathrm{CoV}-2$. Actualmente, en nuestro país, más del $50 \%$ de los niños, niñas y adolescentes viven en la pobreza, con déficits en su alimentación, su salud mental, sus inmunizaciones y su educación. Más que nunca deberemos ejercer una medicina humanizada, con una gran cuota de empatía hacia nuestros pacientes y sus familias.

Los conocimientos actuales, en el concepto de los 1000 días, nos muestran que estos primeros momentos vitales condicionan en gran parte la salud a lo largo de todo el curso de la vida. Esto realza aún más la tarea del pediatra, persuadidos que la verdadera prevención, por ejemplo, de las enfermedades crónicas no transmisibles del adulto (uno de los principales problemas de salud pública a nivel mundial), se inicia en la infancia, y que los consejos y actitudes emanados del pediatra siguen gozando de un gran respeto por parte de la sociedad general.

El distanciamiento social obligatorio ha profundizado y visibilizado los serios problemas educativos de nuestro país, acentuando la gran diferencia de oportunidades entre aquellos con posibilidades de acceder a contenidos curriculares en forma remota y aquellos que por falta de conectividad y/o dispositivos no los han recibido. Esta grave consecuencia educativa se ve aún más complicada con la pérdida de la escuela como ámbito de desarrollo social y contención emocional de nuestros pacientes.

Como sociedad, con la falta de presencialidad escolar, hemos perdido un "capital social" muy importante para la educación y contención de nuestros pacientes, especialmente en los sectores más vulnerables. Esta clara consecuencia social, es más evidente quizás en los y las adolescentes quienes al perder la continuidad de conexión con el ámbito educativo, quedan con una mayor exposición a las problemáticas sociales propias de esta etapa de la vida (violencia en todas sus modalidades, consumo problemático de sustancias, embarazo no deseado, infecciones de transmisión sexual).

Los pediatras nos vemos obligados a reclamar por este espacio fundamental para el correcto desarrollo de nuestra niñez y adolescencia, acompañando a las autoridades sanitarias y educativas en generar las mejores condiciones de seguridad en el ámbito escolar, convencidos que la salud y la educación son dos derechos indivisibles desde el punto de vista pediátrico y que la escuela es el escenario donde mejor se afianza y desarrolla este concepto.

Las tecnologías del conocimiento y la comunicación han logrado un impulso muy importante en estos años de pandemia, impactando en dos aspectos fundamentales de la tarea médica. Han facilitado el acceso a información científica actualizada y la realización en forma remota de distintas actividades de Educación Médica Continua y, desde el punto de vista asistencial, han permitido mantener contacto con los pacientes, en un contexto de distanciamiento social obligatorio. Este último aspecto, si bien ha facilitado la consulta, no debe opacar la mejor calidad de la consulta presencial en el ámbito de la Pediatría general, donde podemos aplicar en forma plena el concepto biopsicosocial en el acompañamiento de niños, niñas y adolescentes y de sus familias, a través del lenguaje verbal y no verbal y de un examen físico apropiado.

Estos desafíos para la Pediatría de los próximos años deberán ser afrontados simultáneamente con la actualización y aplicación asistencial de los nuevos conocimientos generados por la Medicina Traslacional, donde distintas disciplinas trabajan en conjunto para generar nuevas evidencias, para la mejor comprensión de fenómenos biológicos y para acceder a nuevas instancias diagnósticas y terapéuticas. Asimismo, la investigación pediátrica deberá continuar generando los mejores conocimientos y guías para la atención 
de nuestros problemas cotidianos.

Sin dudas, visto desde esta perspectiva integral, la tarea de la Pediatría en nuestro país, en los próximos años, deberá contar con un gran esfuerzo por nuestra parte, pero sin dudas nuestros pacientes y sus familias, quienes esperan lo mejor de nosotros, justifican plenamente continuar trabajando intensamente para honrar el objetivo fundamental de nuestra Sociedad Argentina de Pediatría: "Por una Niñez y Adolescencia sanas en un mundo mejor".

Recordemos, además, que la Pediatría no es solamente una especialidad médica, es "una forma de ser" que nos distingue dentro del universo médico.

\section{Dr. Omar Tabacco}

Presidente

Sociedad Argentina de Pediatría

http: / / dx.doi.org/ 10.5546/ aap.2021.290

Texto completo en inglés:

http: / / dx.doi.org/10.5546/ aap.2021.eng.290

Cómo citar: Tabacco O. La Pediatría argentina en los próximos años. Arch Argent Pediatr 2021;119(5):290-291.

\section{REFERENCIAS}

- Sociedad Argentina de Pediatría. Suplemento COVID-19. Arch Argent Pediatr. 2020. [Consulta: 28 de junio de 2021]. Disponible en: https://www.sap.org.ar/docs/ publicaciones / archivosarg/2020/Suplemento_COVID-19_ completo.pdf

- ComitéNacional deFamilia y Salud Mental, ComitéNacional de Infectología, Comité Nacional de Medicina del Deporte,
Comité Nacional de Pediatría General Ambulatoria, et al. Documento conjunto de posicionamiento para el regreso presencial a las escuelas. Sociedad Argentina de Pediatría. Octubre 2021. [Consulta: 28 de junio de 2021]. Disponible en: https:/ / www.sap.org.ar/uploads/archivos/general/ files_documento-conjunto-escuelas-covid_1602694567.pdf

- Sociedad Argentina de Pediatría, Ministerio de Salud de la Nación. El regreso presencial a la escuela de los niños, niñas y adolescentes con enfermedades crónicas. Orientaciones para los equipos de salud. Marzo 2021. [Consulta: 28 de junio de 2021]. Disponible en: https:/ / www.sap.org.ar/ uploads / archivos / general / files_consideraciones-paravuelta-a-escuelas-03-21_1614956663.pdf

- Cabana JL, Pedra CR, Ciruzzi MS, Garategaray MG, et al. Percepciones y sentimientos de niños argentinos frente a la cuarentena Covid-19. Arch Argent Pediatr. 2021;119(4): S107-22.

- UNICEF. Encuesta de percepción y actitudes de la población. Impacto de la pandemia Covid-19 en las familias con niñas, niños y adolescentes. 2021. [Consulta: 28 de junio de 2021]. Disponible en: https: / / www.unicef.org/argentina/ informes / encuesta-de-percepcion-y-actitudes-de-lapoblacion

- Fondo de las Naciones Unidas para la Infancia. Estudio sobre los efectos de la salud mental de niñas, niños y adolescentes por Covid-19. Buenos Aires: UNICEF; 2021.

- Sociedad Argentina de Pediatría, Dirección de Control de Enfermedades Inmunoprevenibles. Recupero del Calendario de Vacunación. Ministerio de Salud. Mayo 2021. [Consulta: 28 de junio de 2021]. Disponible en: https:/ / www.sap. org.ar / uploads / archivos / general / files_recupero-devacunas-05-21_1620430640.pdf

- Torres F, Domíngez P, Aruanno ME, Macherett MJ, et al. Impacto de la Pandemia porSAR-Cov-2 en la administración de vacunas del Calendario Nacional de Inmunizaciones en menores de 2 años. Arch Argent Pediatr. 2021;119(3):198-201.

- Lachman P. Where to make a difference: research and the social determinants in pediatrics and child health in the COVID-19 era. Pediatr Res. 2021;89(2):259-62.

- Tuñón I, Sánchez ME, García Balus NA, Bauso N. Nuevos retrocesos en las oportunidades de desarrollo de la Infancia y la Adolescencia. Tendencias antes y durante la pandemia COVID-19. Buenos Aires: Educa; 2021. 